\title{
Hepatitis autoinmune en niños. Perspectivas actuales
}

\author{
Autoimmune hepatitis in children. Current perspectives
}

\author{
Dra. Miriam Cuarterolo ${ }^{a}$, Dra. Mirta Ciocca ${ }^{b}$ y Dr. Fernando Álvarez ${ }^{c}$
}

\begin{abstract}
RESUMEN
La hepatitis autoinmune es una enfermedad inflamatoria crónica progresiva del hígado de etiología desconocida. Afecta predominantemente al sexo femenino en la etapa prepuberal. Se caracteriza por presentar niveles elevados de transaminasas e inmunoglobulina $\mathrm{G}$, niveles bajos del factor 4a del complemento y de IgA circulantes, autoanticuerpos en suero, prevalencia del antígenoleucocitariohumano B8, haplotipos DR3 y DR4, y hepatitis de interfase en la histología. El curso puede ser fluctuante, con períodos de remisión espontánea. Se describen dos tipos de acuerdo con los autoanticuerpos hallados en suero. El mecanismo de producción del daño hepático es secundario a reacciones inmunes contra antígenos hepáticos no controladas adecuadamente por las células $\mathrm{T}$ reguladoras. La mayoría de los pacientes responden favorablemente al tratamiento inmunosupresor. Librada a su evolución espontánea, la enfermedad progresa hacia la destrucción hepática, por lo que, en su etapa terminal, requiere un trasplante hepático.

Palabras clave: hepatitis autoinmune, niños, diagnóstico, tratamiento.
\end{abstract}

http:/ /dx.doi.org/10.5546/aap.2014.169

a. Hepatología, Hospital de Pediatría Prof. Dr. Juan P. Garrahan. Buenos Aires, Argentina.

b. Hepatología y Trasplante Hepático Pediátrico, Hospital Alemán. Buenos Aires, Argentina.

c. Departamento de Pediatría, $\mathrm{CHU}$

- Sainte Justine, Universidad de Montreal. Canadá.

Correspondencia:

Dra. Miriam

Cuarterolo:

mcuarterolo@intramed. net

Conflicto de intereses: Ninguno que declarar.

Recibido: 7-1-2014

Aceptado: 8-1-2014

\section{INTRODUCCIÓN}

La hepatitis autoinmune (HAI) es una enfermedad inflamatoria crónica progresiva del hígado de etiología desconocida. Afecta predominantemente al sexo femenino en la etapa prepuberal. Se caracteriza por presentar niveles elevados de transaminasas e inmunoglobulina G (Ig G), niveles bajos del factor $4 a$ del complemento ( $\mathrm{C} 4 \mathrm{a}$ ) y de $\operatorname{Ig} \mathrm{A}$ circulantes, autoanticuerpos en suero, prevalencia del antígeno leucocitario humano (HLA) B8, haplotipos DR3 y DR4, y hepatitis de interfase en la histología. El curso puede ser fluctuante, con períodos de remisión espontánea. Se describen dos tipos de acuerdo con los autoanticuerpos hallados en suero. La hepatitis tipo 1 se caracteriza por presentar anticuerpos antimúsculo liso (SMA), antiactina y/o antinúcleo (ANA) y/o antiantígeno soluble hepático (SLA). En la HAI tipo 2, se encuentran anticuerpos antimicrosomas de hígado y riñón (LKM1) y/o anticitosol hepático tipo 1 (LC1). ${ }^{1-3}$

El mecanismo de producción del daño hepático es secundario a reacciones inmunes contra antígenos hepáticos no controladas adecuadamente por las células $\mathrm{T}$ reguladoras. La mayoría de los pacientes responde favorablemente al tratamiento inmunosupresor. Librada a su evolución espontánea, la enfermedad continúa hacia la destrucción hepática, por lo que, en su etapa terminal, requiere un trasplante hepático $(\mathrm{TH})$.

\section{Genética y patogenia}

La HAI no sigue un patrón mendeliano de herencia; no se ha identificado ningún locus genético definido responsable de la enfermedad. Se describe que uno o más genes actuarían solos o en conjunto, reduciendo o aumentando la susceptibilidad. La mayoría están ubicados en la región del brazo corto del cromosoma 6 del antígeno HLA; la mayor asociación se encuentra dentro del locus DRB1., ${ }^{4}$

Otros genes localizados en el HLA se vinculan con la susceptibilidad para la HAI, tales como el de la IgA y el del factor C4a. La enfermedad puede asociarse, por consiguiente, con deficiencia de IgA y con bajos niveles de C4a. ${ }^{6}$

Genes fuera del locus HLA han sido vinculados con la HAI; estos codifican proteínas con influencias en los sistemas inmunes innato o adquirido. Los siguientes polimorfismos fueron vinculados con 
la predisposición a desarrollar HAI: antígeno 4 de linfocitos T citotóxicos (CTLA-4), receptor de vitamina $\mathrm{D}$, factor- $\alpha$ de necrosis tumoral (TNF- $\alpha$ ). Mutaciones en el gen regulador autoinmune (AIRE), responsables del desarrollo de distrofia ectodérmica-candidiasis-poliendocrinopatía (APECED), pueden asociarse a la HAI en el 10$20 \%$ de los casos. ${ }^{7-10}$

La mayoría de las enfermedades autoinmunes se desarrollan más frecuentemente en el sexo femenino. En la HAI, la relación mujeres:varones es de 3:1 en la tipo 1 y de 9:1 en la tipo 2. Existen diferencias en la respuesta inmune básica entre mujeres y varones; por ejemplo, luego de la vacunación, las mujeres presentan niveles más elevados de anticuerpos y mayor activación de células T. Además, se ha demostrado que los estrógenos aumentan in vitro la producción de citoquinas Th1 por los linfocitos $\mathrm{T}$, mientras que los andrógenos la disminuyen.

La edad también influye en la incidencia de algunas enfermedades autoinmunes, lo que sugiere una relación hormonal en su patogenia. La HAI es predominantemente una enfermedad pediátrica; el $40 \%$ de la tipo 1 y el $80 \%$ de la tipo 2 son diagnosticados antes de la edad de 18 años. El pico de incidencia de la enfermedad en la infancia se presenta en la etapa prepuberal, mientras que, en la adultez, ocurre en mujeres luego de la menopausia.

Factores ambientales actuarían como disparadores del desarrollo de HAI en individuos predispuestos genéticamente, de edad y sexo particular. Estos factores incluyen drogas, productos químicos o virus. Se considera que son capaces de promover la respuesta autoinmune por intermedio de los siguientes mecanismos: activación inespecífica de células $T$ en reposo, modificación o liberación de proteínas secuestradas, reacción cruzada entre un virus y una proteína propia del paciente (mecanismo de semejanza molecular) y modulación de la expresión genética. ${ }^{1,11-14}$

\section{DIAGNÓSTICO \\ Hallazgos clínicos}

La hepatitis aguda es la forma más habitual de presentación en la población pediátrica e infrecuentemente puede acompañarse con insuficiencia hepática. La enfermedad puede presentarse también como hepatitis crónica. Algunos pacientes manifiestan solo síntomas inespecíficos, como anorexia, pérdida de peso y decaimiento, que dificultan la realización de un diagnóstico temprano. En un 10-15\% de los casos, los hallazgos de hepatomegalia, esplenomegalia o un aumento de transaminasas inexplicable constituyen las únicas manifestaciones de la enfermedad. Las edades medias de presentación son 10 años y 6,5 años para las HAI tipo 1 y 2, respectivamente (Tabla 1 ).

En el examen físico, se observa ictericia, estigmas de cronicidad (angiomas estelares y eritema palmar) y hepatomagalia. Cuando la enfermedad avanza sin ser diagnosticada, son frecuentes los hallazgos compatibles con hipertensión portal, como circulación colateral abdominal, esplenomegalia, aumento de la relación epiplón-aorta en la ecografía, colaterales venosas, así como la presencia de várices esofágicas y gastropatía hipertensiva observadas en la endoscopía. La heterogeneidad de las formas de presentación y el curso fluctuante de la enfermedad, con períodos secuenciales de actividad y remisión espontánea, son elementos que pueden retrasar el diagnóstico, por lo que la enfermedad sin tratamiento lleva a la destrucción hepática. Los pacientes con complicaciones de la cirrosis, como hemorragia

Tabla 1. Hepatitis autoinmune. Formas de presentación - Diagnósticos diferenciales

Síndrome de hepatitis aguda (más frecuente)

Hepatitis fulminante o subfulminante

Hepatitis crónica

Síntomas inespecíficos (decaimiento, anorexia)

Hepatomegalia y/o esplenomegalia en examen de rutina, con aumento de transaminasas
Hepatitis viral aguda: hepatitis A, B, E, CMV, VEB, herpes simple, parvovirus B19, adenovirus, colangitis esclerosante primaria, enfermedad de Wilson.

Hepatitis A, B, CMV, VEB, parvovirus B19, indeterminada, enfermedad de Wilson.

Deficiencia de alfa 1 antitripsina, hepatitis tóxica (minociclina), colangitis esclerosante primaria, enfermedad de Wilson.

Enfermedad celíaca, deficiencia de alfa 1 antitripsina, hepatitis viral anictérica, enfermedad de Wilson.

Hepatitis viral anictérica, deficiencia de alfa 1 antitripsina, enfermedad de Wilson. 
digestiva intratable, ascitis, peritonitis bacteriana espontánea, síndrome hepatopulmonar, tienen indicación de $\mathrm{TH}$, así como los que se presentan con fallo hepático agudo y encefalopatía. Las enfermedades autoinmunes extrahepáticas se asocian en el $40 \%$ de los pacientes y en el 35\% de los familiares de primer orden. En la HAI tipo 1: colitis ulcerosa, enfermedad de Crohn, vasculitis, trombocitopenia, anemia hemolítica, glomerulonefritis, alveolitis fibrosante; en la tipo 2: enteropatía autoinmune, tiroiditis, diabetes, vitíligo, síndrome de distrofia ectodérmica, candidiasis y poliendrocrinopatía autoinmune, alopecía, síndrome linfoproliferativo autoinmune. La enfermedad celíaca puede estar asociada más frecuentemente a la HAI tipo $1 .{ }^{15,16}$

\section{Laboratorio}

El aumento de los niveles de transaminasas, entre 2 y 50 veces los valores normales, la hipergammaglobulinemia, secundaria a la proliferación inespecífica de linfocitos B, la hiperbilirrubinemia y los niveles normales de gammaglutamil transpeptidasa o levemente aumentados son hallazgos característicos. El déficit de $\operatorname{Ig} \mathrm{A}<1,2 \mathrm{~g} / 1$ se encuentra en el $45 \%$ de la HAI tipo 2 y en el $9 \%$ de la tipo 1; los niveles de C4a pueden estar disminuidos hasta en el 69\% de los casos. En pacientes con cirrosis e hipertensión portal, se observa leucopenia y pancitopenia secundarias a hiperesplenismo; aquellos con insuficiencia hepática presentan disminución de los niveles de albúmina y de factores de la coagulación.

Los autoanticuerpos circulantes poseen valor diagnóstico y permiten diferenciar los dos tipos de HAI. Los ANA (patrón homogéneo) y el SMA, característicos de la HAI tipo 1, y el LKM1, característico de la HAI tipo 2, rara vez se hallan simultáneamente. Se detectan por inmunofluorescencia indirecta sobre preparados de riñón, hígado y células gástricas. Los títulos clínicamente relevantes para el diagnóstico de HAI en la población pediátrica son 1/20 para ANA y SMA, y 1/10 para LKM1. Los anticuerpos ANA y SMA no son específicos de la HAI tipo 1 y pueden hallarse en otras enfermedades, como las hepatitis crónicas virales B y C. El LKM1 es un anticuerpo altamente específico de la HAI tipo 2 y se ha encontrado en una proporción menor del 5\% de pacientes con hepatitis C crónica. En un bajo porcentaje de casos, los autoanticuerpos pueden ser negativos. Otros anticuerpos menos frecuentemente determinados pero de importancia diagnóstica en la HAI pediátrica incluyen el LC1, anticitoplasma de neutrófilos (PANCA) y el SLA. El LC1 suele estar asociado con el LKM1, lo que constituye un marcador adicional para la HAI tipo 2. El PANCA puede observarse asociado a la HAI tipo 1, enfermedad inflamatoria intestinal y colangitis esclerosante primaria. El SLA, originalmente descrito como marcador de un tercer tipo de HAI, se encuentra en alrededor del $50 \%$ de los pacientes con HAI tipo 1 y 2, lo que caracteriza un curso evolutivo más grave (Tabla 2$).{ }^{15-18}$

\section{Histología}

La realización de la biopsia hepática aporta elementos para la confirmación diagnóstica y permite, además, evaluar la gravedad del daño hepático. En pacientes con alteraciones de la coagulación en el momento del diagnóstico, la contraindicación de realizar la biopsia no debe retrasar el comienzo del tratamiento.

El hallazgo histológico característico de la HAI es la presencia de un infiltrado linfoplasmocítico en el espacio porta, integrado por linfocitos T y B,

TABLA 2. Hepatitis autoinmune. Tipos y autoanticuerpos

\begin{tabular}{lccc}
\hline Autoanticuerpo & HAI tipo 1 & HAI tipo 2 & Antígeno \\
\hline SMA & $90-100 \%$ & & Filamentos de actina \\
ANA & $0-10 \%$ & & Varios \\
SMA / ANA & $40-60 \%$ & $40-50 \%$ & Citocromo P450 2D6 \\
LKM1 & & $10-15 \%$ & Formiminotransferasa ciclodeaminasa \\
LC1 & & $35-45 \%$ & tRNP \\
LKM1/LC1 & & Receptor de asialoglicoproteína \\
SLA & $50 \%$ & & \\
ASGP-R & $75 \%$ &
\end{tabular}

HAI: hepatitis autoinmune. SMA: anticuerpos antimúsculo liso. ANA: anticuerpos antinucleares. LKM1: anticuerpos antimicrosomas de hígado y riñón tipo 1. LC1: anticuerpos anticitosol hepático tipo 1. SLA: antiantígeno soluble hepático. ASGP-R: receptor de la asialoglicoproteína. tRNP: ribonucleoproteínas de transferencia. 
macrófagos y células plasmáticas, que invade el lóbulo hepático. Este infiltrado y la destrucción de los hepatocitos ubicados en la periferia del lóbulo, con erosión de la placa limitante, ocasionan la denominada hepatitis de interfase. La necrosis en puente y la formación de rosetas de hepatocitos se observan también con frecuencia. El hallazgo de cirrosis se encuentra hasta en un tercio de los pacientes en el momento del diagnóstico, independientemente del modo de presentación de la enfermedad, lo que indica una evolución subclínica previa prolongada. La presentación con falla hepática aguda puede mostrar una histología de necrosis masiva y colapso multilobular con leve fibrosis portal (Figuras 1, 2 y 3). ${ }^{19}$

\section{Diagnósticos diferenciales}

Se deben descartar las hepatitis crónicas virales B y C, la enfermedad de Wilson y la ingesta de tóxicos. En pacientes con presentación aguda, deben excluirse, además, otros virus hepatotropos, como el de las hepatitis A y E, citomegalovirus, virus de Epstein-Barr, herpes simple, parvovirus B19 y adenovirus (Tabla 1).

\section{Criterios diagnósticos del Grupo Internacional para el Estudio de la Hepatitis Autoinmune}

Existe un sistema de puntaje propuesto por el Grupo Internacional para el Estudio de la Hepatitis Autoinmune (International Autoimmune Hepatitis Group, IAHG), desarrollado con fines científicos. ${ }^{20}$ Posteriormente, el IAHG ha presentado una simplificación de este sistema. ${ }^{21}$

FIGURA 1. Hematoxilina y eosina 100X. Espacio porta con hepatitis de interfase linfoplasmocitaria de moderada a marcada

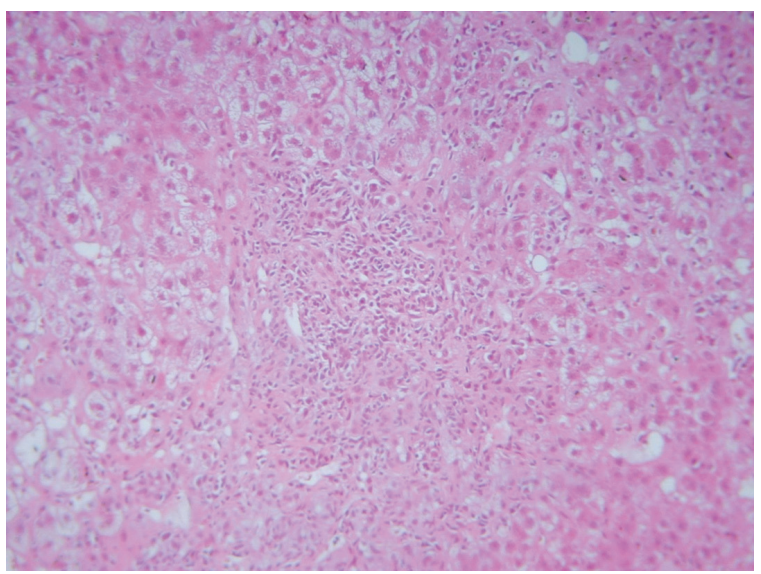

\section{Síndromes de superposición}

Los pacientes presentan compromiso de los conductos biliares sugestivos de colangitis esclerosante y hallazgos de HAI. El diagnóstico diferencial se establece con estudios de imágenes de la vía biliar, como la colangiografía por resonancia magnética. ${ }^{15}$

\section{Tratamiento y pronóstico}

El tratamiento tiene como objetivo el control del proceso inflamatorio del parénquima hepático, obtener la remisión clínica y bioquímica de la enfermedad, definida por niveles normales de transaminasas y gammaglobulina, y

FIgURA 2. Hematoxilina y eosina 400X. Sector de interfase con inflamación linfoplasmocitaria y necrosis de hepatocitos con cambios degenerativos asociados y esteatosis focal

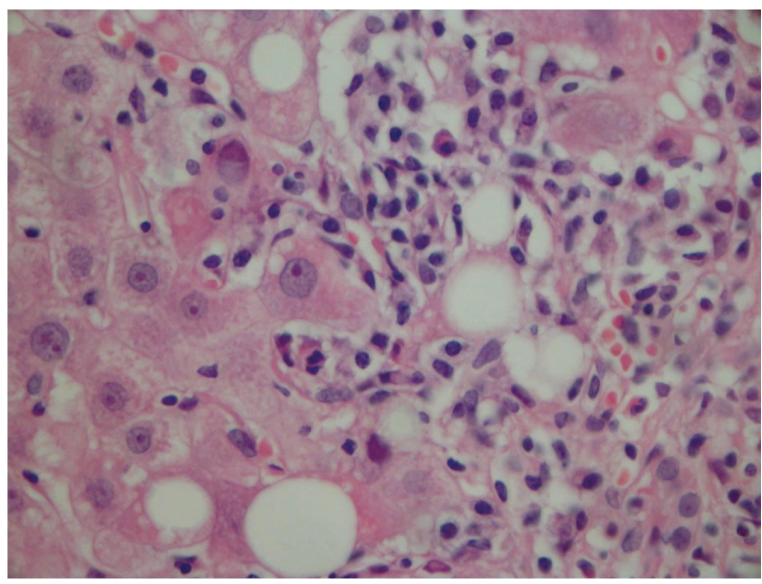

Figura 3. Tricrómico 25X. La tinción de tricrómico destaca la presencia de puentes fibrosos porto-portales. Coexiste infiltrado inflamatorio mononuclear en los tractos fibrosos y espacio porta remanentes

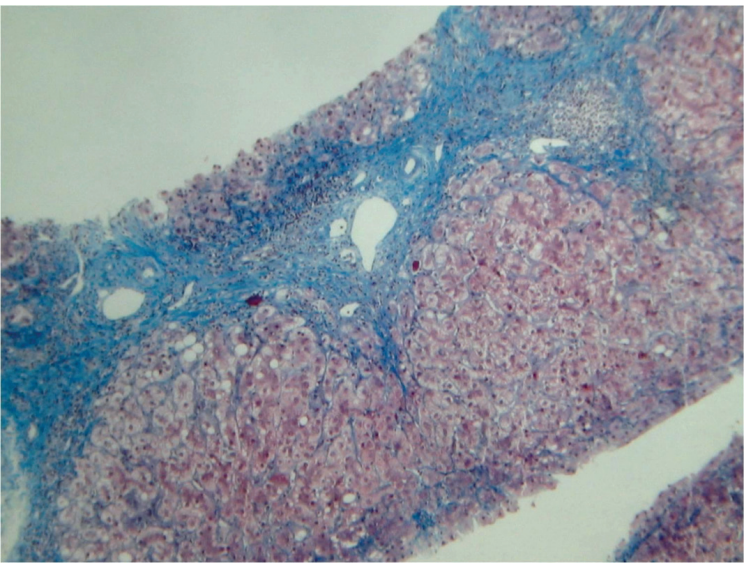


su mantenimiento con mínimas dosis de inmunosupresores. La respuesta al tratamiento inmunosupresor es mayor del $80 \%$ e incluye aquellos casos que se presentan con insuficiencia hepática (tiempo de protrombina $<50 \%$ postadministración de vitamina $\mathrm{K}$ ), con excepción de aquellos pacientes que se presentan con encefalopatía. El primer esquema terapéutico implementado consiste en la asociación de prednisona y azatioprina. La dosis inicial de prednisona es de $2 \mathrm{mg} / \mathrm{kg} /$ día (dosis máxima: $60 \mathrm{mg} /$ día) y de azatioprina, 1,5-2 mg/ kg/ día. Los esteroides se reducen gradualmente a medida que se produce el descenso de los niveles de transaminasas, lo que ocurre durante los primeros dos meses de tratamiento en la mayoría de los pacientes. Obtenida la remisión de la enfermedad, se indican los mínimos niveles de inmunosupresión capaces de mantenerla. Los efectos adversos relacionados con la administración prolongada de esteroides son obesidad, hábito cushingoide, estrías cutáneas, hipertricosis, osteopenia/osteoporosis, diabetes, cataratas, hipertensión arterial, psicosis.

Las complicaciones de la azatioprina son infrecuentes e incluyen colestasis, enfermedad venooclusiva, pancreatitis, náuseas, vómitos, rash y toxicidad de médula ósea. ${ }^{15,22-24}$
En aquellos casos intolerantes o no respondedores al tratamiento convencional, se han propuesto drogas alternativas.

La ciclosporina es un inmunosupresor potente que se ha utilizado efectivamente tanto en niños como en adultos con HAI. En la edad pediátrica, se ha demostrado que la ciclosporina indicada como droga de ataque puede inducir la remisión en HAI tipo 1 y 2. Si bien los efectos adversos de este fármaco pueden ser importantes, con los niveles que ha sido utilizado y durante períodos breves, tres meses en la mayoría de los pacientes, resultaron leves y transitorios ${ }^{25,26}$ (Tabla 3).

El micofenolato de mofetilo ha sido empleado en un pequeño grupo de pacientes pediátricos con buenos resultados. La realización de una curva de farmacocinética de la droga, lo que es relativamente complicado, es la única manera de establecer la dosis adecuada. Los efectos adversos pueden ser graves. ${ }^{27,28}$

Existen publicaciones anecdóticas sobre agentes anti-TNF, como el infliximab. ${ }^{29}$

Recientemente, se ha publicado una experiencia exitosa con rituximab en dos casos refractarios a otras drogas alternativas. ${ }^{30}$

La budesonida (dosis de $3 \mathrm{mg}$, 3 veces por día) más azatioprina ha sido utilizada en comparación con la asociación de prednisona (40 mg/día) más

TABLA 3. Hepatitis autoinmune. Esquemas de tratamiento de acuerdo con la intensidad de la inflamación hepática

\begin{tabular}{lccc}
\hline & & & HAI juvenil o LKM1 (+) \\
\hline RIN & $\mathrm{N}$ & $<1,5$ & $>1,5$ \\
Albúmina & $\mathrm{N}$ & $3 \mathrm{~g} / \mathrm{l}$ & $<3 \mathrm{~g} / 1$ \\
Bilirrubina & $\mathrm{N}$ & $<3 \times \mathrm{N}$ & $>3 \times \mathrm{N}$ \\
Transaminasas & $\leq 1,5 \times \mathrm{N}$ & $>2-8 \times \mathrm{N}$ & $>8 \times \mathrm{N}$ \\
Inmunosupresión & Baja & Moderada & Elevada \\
Esquema convencional & Prednisona: & Prednisona: & Prednisona: \\
& $0,3-0,5 \mathrm{mg} / \mathrm{kg} / \mathrm{d}$ & $1-2 \mathrm{mg} / \mathrm{kg} / \mathrm{d}$ & $1-2 \mathrm{mg} / \mathrm{kg} / \mathrm{d}$ \\
& & $($ máximo: $60 \mathrm{mg} / \mathrm{d})$ & Azatioprina: \\
& Azatioprina: & Azatioprina: & $1,5-2 \mathrm{mg} / \mathrm{kg} / \mathrm{d}$ \\
Ciclosporina neoral & $1,5 \mathrm{mg} / \mathrm{kg} / \mathrm{d}$ & $1,5-2 \mathrm{mg} / \mathrm{kg} / \mathrm{d}$ & $\mathbf{y}$ \\
& & $\mathbf{0}$ & $\mathrm{Nivel} \mathrm{C} 0:$
\end{tabular}

RIN: relación internacional normalizada. HAI: hepatitis autoinmune. LKM1: anticuerpos antimicrosomas de hígado y riñón tipo 1. Controles semanales. Se indica disminución progresiva de las dosis de inmunosupresores según respuesta (mejoría de los niveles de transaminasas, normalización del RIN).

La ciclosporina neoral se suspende una vez obtenida la remisión de la enfermedad y se continúa con dosis mínimas de prednisona + azatioprina. 
azatioprina como esquemas de ataque durante 6 meses en un trabajo que incluyó a 46 niños y adolescentes. Posteriormente, todos los pacientes recibieron budesonida más azatioprina durante los siguientes 6 meses. La tasa de remisión obtenida con ambos esquemas después de los 6 meses de tratamiento inicial ( $16 \%$ y $15 \%$ ) y luego de 12 meses de budesonida más azatioprina (50\% y $42 \%)$, respectivamente, fue menor a la observada con la asociación convencional de prednisona ( $2 \mathrm{~m} / \mathrm{kg}$ /día, máximo: $60 \mathrm{mg} /$ día) más azatioprina. Los efectos adversos fueron similares con ambos esquemas, y se observó mayor aumento de peso en los pacientes bajo prednisona. Si bien la asociación budesonidaazatioprina puede ser una alternativa en pacientes con HAI sin cirrosis, no existen evidencias que permitan indicar que este esquema constituye un tratamiento de primera línea para la HAI. . $^{31,32}$

Es posible intentar la suspensión del tratamiento inmunosupresor luego de la obtención y persistencia de la remisión por un tiempo prolongado, no menor de dos años; sin embargo, no existe ninguna publicación de pacientes pediátricos seguidos prospectivamente que confirme o desestime esta proposición. Se menciona una posible discontinuación de la terapia en el $20 \%$ de los casos de la HAI tipo 1, pero en ningún niño con la tipo $2.33,34$

El TH es el recurso terapéutico de elección para aquellos casos que desarrollan enfermedad hepática avanzada a pesar del tratamiento inmunosupresor y para los que presentan un fallo hepático fulminante. La sobrevida del paciente trasplantado por esta enfermedad es de $86 \%$ a los 5 años. ${ }^{15,33-38}$ La HAI puede recurrir luego del TH hasta en un $30 \%$ de los casos.

\section{Perspectivas futuras}

Existen numerosas publicaciones de los últimos años sobre reconstrucción de la autotolerancia a través de intervenciones inmunológicas específicas. ${ }^{39,41}$

\section{CONCLUSIONES}

El diagnóstico de HAI debe considerarse en pacientes con signos y síntomas de hepatitis aguda o crónica. El curso fluctuante de la enfermedad puede determinar la alternancia de períodos de actividad y remisión, lo que genera dificultades para realizar el diagnóstico. El establecimiento temprano del diagnóstico y la terapéutica apropiada evitan, en la mayoría de los casos, la progresión de la enfermedad y el requerimiento de $\mathrm{TH}$. El manejo adecuado de la inmunosupresión y el seguimiento minucioso de los pacientes permiten el control de la enfermedad en el largo plazo con mínimos efectos adversos. El logro de autotolerancia luego de varios años de tratamiento es posible en algunos pacientes. Nuevos estudios están focalizados en obtener herramientas terapéuticas que permitan la reconstrucción de la autotolerancia.

\section{Agradecimientos}

A los Dres. Marcelo Fabián Amante y Gabriel Casas, Servicio de Anatomía Patológica del Hospital Alemán, de Buenos Aires.

\section{BIBLIOGRAFÍA}

1. Béland $K$, Lapierre $P$, Alvarez F. Influence of genes, sex, age an environment on the onset of autoimmune hepatitis. World J Gastroenterol 2009;15(9):1025-34.

2. Mieli-Vergani G, Heller S, Jara P, Vergani D, et al. Autoimmune hepatitis. I Pediatr Gastroenterol Nutr 2009;49(2):158-64.

3. Mieli-Vergani G, Vergani D. Autoimmune hepatitis. Nat Rev Gastroenterol Hepatol 2011;8:320-9.

4. DonaldsonPT.Genetics of theliverdisease:Immunogenetics and Disease Pathogenesis. Gut 2004;53:599-608.

5. Fainboim L,Marcos Y,PandoM,CapucchioM, etal.Chronic active autoimmune hepatitis in children. Strong association with a particular HLA-DR6 (DRB1*1301) haplotype. Hum Immunol 1994;41:146-50.

6. Gregorio GV, Portmann B, Reid F, Donaldson PT, et al. Autoimmune hepatitis in children: a 20-year experience. Hepatology 1997;25:541-7.

7. Djilali-Saiah I, Ouellette P, Caillat-Zucman S, Debray D, et al. CTLA-4/CD 28 region polymorphisms in children from families with autoimmune hepatitis. Hum Immunol 2001;62:1356-62.

8. Vogel A, Strassburg CP, Manns MP. Genetic association of vitamin D receptor polymorphisms with primary biliary cirrhosis and autoimmune hepatitis. Hepatology 2002;35:126-31.

9. Czaja AJ, Cookson S, Constantini PK, Clare M, et al. Cytokine polymorphisms associated with clinical features and treatment outcome in type 1 autoimmune hepatitis. Gastroenterology 1999;117:645-52.

10. Ahonen P, Myllarniemi S, Sipila I, Perheentupa J. Clinical variation of autoimmune polyendocrinopathy-candidiasisectodermal dystrophy (APECED) in a series of 68 patients. N Engl J Med 1990;322:1829-36.

11. Longhi MS, Ma Y,Mieli-Vergani G, Vergani D. Regulatory T cells in autoimmune hepatitis. J Hepatol 2012;57(4):932-3.

12. Oo YH, Adams DH. Regulatory T cells and autoimmune hepatitis: defective cells or a hostile environment? J Hepatol 2012;57(1):6-8.

13. Lapierre P, Beland K, Martin C, Alvarez Jr F, Alvarez F. Forkhead box $\mathrm{p} 3 \mathrm{p}$ regulatory $\mathrm{T}$ cell underlies male resistance to experimental type 2 autoimmune hepatitis. Hepatology 2010;51:1789-98.

14. Liberal R, Grant CR, Mieli-Vergani G, Vergani D. Autoimmune hepatitis: A comprehensive review. J Autoimmun 2013;41:126-39.

15. Alvarez F. Autoimmune hepatitis and primary sclerosing colangitis. Clin Liv Dis 2006;89-107.

16. Mieli-Vergani G, Vergani D. Autoimmune hepatitis. Nat Rev Gastroenterol Hepatol 2011;8:320-9.

17. Bogdanos DP, Invernizzi P, Mackay IR, Vergani D. 
Autoimmune liver serology: Current diagnostic and clinical challenges. World J Gastroenterol 2008;14: 3374-87.

18. DonaldsonPT.Genetics of theliver disease:immunogenetics and disease pathogenesis. Gut 2004;53:599-608.

19. Stravitz RT, Lefkowitch JH, Fontana RJ, Gershwin ME, et al. Autoimmune acute liver failure: proposed clinical and histological criteria. Hepatology 2011;53:517-26.

20. AlvarezF, Berg PA, Bianchi FB, Bianchi L, et al. International Autoimmune Hepatitis Group Report: review of criteria for diagnosis of autoimmune hepatitis. J Hepatol 1999;31:929-38.

21. Hennes EM, Zeniya M, Czaja AJ, Pares A, et al. Simplified criteria for the diagnosis of autoimmune hepatitis. Hepatology 2008;48:169-76.

22. VerganiD, Mieli-VerganiG. Pharmacological management of autoimmune hepatitis. Expert Opin Pharmacother 2011;12:607-13.

23. Manns MP, Czaja AJ, Gorham JD, Krawitt EL, et al. Diagnosis and management of autoimmune hepatitis. Hepatology 2010;51:2193-e213.

24. Cuarterolo ML, Ciocca ME, López SI, De Dávila MT, Alvarez F. Immunosuppressive therapy allows recovery from liver failure in children with autoimmune hepatitis. Clin Gastroenterol Hepatol 2011;9(2):145-9.

25. Alvarez F, Ciocca M, Cañero-Velasco M, Ramonet M, et al. Short-term cyclosporine induces a remission of autoimmune hepatitis in children. J Hepatol 1999;30(2):222-7.

26. CuarteroloM,Ciocca M,CañeroC, RamonetM, etal. Followup of children with autoimmune hepatitis in children. $J$ Pediatr Gastroenterol Nutr 2006;43:635-9.

27. Aw MM, Dhawan A, Samyn M, Bargiota A, et al. Mycophenolatomofetil as rescue treatment for autoimmune liver disease in children: A 5-year follow-up. J Hepatol 2009;51:156-60.

28. Halac U, Álvarez F. New hope for difficult cases of autoimmune hepatitis. Nature Reviews 2009;6:629-30.

29. Weiler-Normann C, Wiegard C,Schramm C, Lohose AW.A case of difficult-to-treat autoimmune hepatitis successfully managed by TNF-alpha blockade. Am J Gastroenterol 2009;104:2877-8.
30. D'Agostino D, Costaguta A, AlvarezF. Successful treatment of refractory autoimmune hepatitis with rituximab. Pediatrics 2013;132(2):526-30.

31. Woynarowski M, Nemeth A, Baruch Y, Koletzko S, et al. On behalf of the European Autoimmune HepatitisBudesonide Study Group. Budesonide versus prednisone with azathioprine for the treatment of autoimmune hepatitis in children and adolescents. J Pediatr 2013;163:1347-57.

32. Mieli-Vergani G, Vergani D. Budesonide for juvenile autoimmune hepatitis? Not yet. JPediatr 2013;163(5):1246-8.

33. Murray KF, Shah U, Mohan N, Heller S, et al. Chronic hepatitis. J Pediatr Gastroenterol Nutr 2008;47:225-33.

34. Greene MT, Whitington PF. Outcomes in pediatric autoimmune hepatitis. Curr Gastroent Rep 2009;11:248-51.

35. Gregorio GV, Portmann B, Reid F, Donaldson PT, et al. Autoimmune hepatitis in childhood: a 20-year experience. Hepatology 1997;25(3):541-7.

36. Martin S, Alvarez F, Anand R, Song C, et al. Outcomes in children who underwent transplantation for autoimmune hepatitis. Liver transplantation 2011;17:393-401.

37. Manns MP, Czaja AJ, Gorham JD, Krawitt EL, et al. Diagnosis and management of autoimmune hepatitis. Hepatology 2010;51:2193-213.

38. Chai PF, Lee W, Brown Y, McPartland J, et al. Childhood autoimmune liver disease: indications and outcome of liver transplantation. J Pediatr Gastroenterol Nutr 2010;50:295-302.

39. Lapierre P, Beland K, Yang R, Alvarez F. Adoptive transfer of exvivo expanded regulatory $\mathrm{T}$ cells in an autoimmune hepatitis murine model restores peripheral tolerance. Hepatology 2013;57(1):217-27.

40. Beland K, LapierreP, Djilali-Saiah I, Alvarez F. Liver restores immune homeostasis after local inflammation despite the presence of autoreactive T cells. PLOS ONE 7(10): e48192. doi:10.1371/journal.pone.0048192.

41. Longhi MS, Hussain MJ, Kwok WW, Mieli-Vergani G, et al. Autoantigen-specific regulatory $\mathrm{T}$ cells, a potential tool for immune-tolerance reconstitution in type-2 autoimmune hepatitis. Hepatology 2011;53:536-47. 